\title{
Improved kinematics for brown dwarfs and very low-mass stars in Chamaeleon I and a discussion of brown dwarf formation ${ }^{\star}$
}

\author{
V. Joergens
}

Leiden Observatory / Sterrewacht Leiden, PO Box 9513, 2300 RA Leiden, The Netherlands

e-mail: viki@strw.leidenuniv.nl

Received 12 April 2005 / Accepted 14 September 2005

\section{ABSTRACT}

We present a precise kinematic study of very young brown dwarfs in the Cha I cloud based on radial velocities (RVs) measured with UVES at the VLT. The kinematics of the brown dwarfs in Cha I are compared to the kinematics of T Tauri stars in the same field, based on both UVES measurements for very low-mass ones and on $R V \mathrm{~s}$ from the literature. More UVES spectra were taken compared with a former paper (Joergens \& Guenther 2001, A\&A, 379, L9), and the reduction of the spectra was improved, while studying the literature for $R V \mathrm{~s}$ of T Tauri stars in Cha I led to a cleaned and enlarged sample of $\mathrm{T}$ Tauri stars. The result is an improved empirical $R V$ distribution of brown dwarfs, as well as of $\mathrm{T}$ Tauri stars in Cha I. We found that the $R V \mathrm{~s}$ of the nine brown dwarfs and very low-mass stars (M6-M 8) in ChaI that were studied have a mean value of $15.7 \mathrm{~km} \mathrm{~s}^{-1}$ and a dispersion measured in terms of a standard deviation of $0.9 \mathrm{~km} \mathrm{~s}^{-1}$, and they cover a total range of $2.6 \mathrm{~km} \mathrm{~s}{ }^{-1}$. The standard deviation is consistent with the dispersion measured earlier in terms of fwhm of $2.1 \mathrm{~km} \mathrm{~s}^{-1}$. The studied sample of $25 \mathrm{~T}$ Tauri stars (G2-M 5) has a mean $R V$ of $14.7 \mathrm{~km} \mathrm{~s}^{-1}$, a dispersion in terms of standard deviation of $1.3 \mathrm{~km} \mathrm{~s}^{-1}$ and in terms of fwhm of $3.0 \mathrm{~km} \mathrm{~s}$, and a total range of $4.5 \mathrm{~km} \mathrm{~s}^{-1}$. The $R V$ dispersion of the brown dwarfs is consistent within the errors with that of T Tauri stars, which is in line with the finding of no mass dependence in some theoretical models of the ejection-scenario for the formation of brown dwarfs. In contrast to current $N$-body simulations, we did not find a high-velocity tail for the brown dwarfs $R V \mathrm{~s}$. We found hints suggesting different kinematics for binaries compared to predominantly single objects in Cha I, as suggested by some models. The global $R V$ dispersion for Cha I members $\left(1.24 \mathrm{~km} \mathrm{~s}^{-1}\right)$ is significantly lower than for Taurus members $\left(2.0 \mathrm{~km} \mathrm{~s}^{-1}\right)$, despite higher stellar density in Cha I showing that a fundamental increase in velocity dispersion with stellar density of the star-forming region is not established observationally. The $R V \mathrm{~s}$ of brown dwarfs observed in Cha I are less dispersed than predicted by existing models for the ejection-scenario.

Key words. stars: low-mass, brown dwarfs - techniques: radial velocities - stars: pre-main sequence - stars: formation

\section{Introduction}

The formation of objects below or close to the hydrogen burning limit is one of the main open issues in the field of the origins of solar systems. The almost complete absence of brown dwarfs in close ( $<3 \mathrm{AU})$ orbits around solarlike stars ("brown dwarf desert") found in ongoing high-precision $R V$ surveys compared to the detection of more than 150 extrasolar planets in this separation range (e.g. Moutou et al. 2005; Marcy et al. 2005) suggests that brown dwarfs generally do not form like planets by dust condensation in a circumstellar disk. On the other hand, a starlike formation from direct gravitational collapse and fragmentation of molecular clouds requires the existence of cloud cores that are cold and dense enough to become Jeans-unstable for brown dwarf masses, which have not yet been found. On theoretical grounds, the opacity limit for

\footnotetext{
* Based on observations at the Very Large Telescope of the European Southern Observatory at Paranal, Chile in program 65.L-0629, 65.I-0011, 268.D-5746, 72.C-0653.
}

the fragmentation (Low \& Lynden-Bell 1976) might prevent the formation of (lower mass) brown dwarfs by direct collapse.

An alternative scenario was proposed in recent years, namely the formation of brown dwarfs by direct collapse of unstable cloud cores of stellar masses that would have become stars if the accretion was not stopped at an early stage by an external process before the object had accreted to stellar mass. It was proposed that such an external process could be the ejection of the protostar out of the dense gaseous environment due to dynamical interactions (Reipurth \& Clarke 2001), analogous to the formation of so-called run-away T Tauri stars (Sterzik \& Durisen 1995, 1998; Durisen et al. 2001). It is known that the dynamical evolution of gravitationally interacting systems of three or more bodies leads to frequent, close two-body encounters and to the formation of close binary pairs out of the most massive objects in the system, as well as to the ejection of the lighter bodies into extended orbits or out of the system with escape velocity (e.g. Valtonen \& Mikkola 1991). The escape of the lightest body is an expected outcome, since the escape probability scales approximately as the inverse third power of 
the mass. The suggestion of this embryo-ejection model as the formation mechanism for brown dwarfs has stimulated in past years hydrodynamical collapse calculations (Bate et al. 2002, 2003; Bate \& Bonnell 2005) and numerical $N$-body simulations (Sterzik \& Durisen 2003; Delgado-Donate et al. 2003, 2004; Umbreit et al. 2005) which predict observable properties of brown dwarfs formed in this way. There are significant differences in the theoretical approaches and predictions of these models, which will be discussed later (see also Joergens 2005 for a recent review of brown dwarf formation).

An external process that prevents the stellar embryo from further accretion and growth in mass can also be photoevaporation by a strong UV wind from a nearby hot O or B star (Kroupa \& Bouvier 2003; Whitworth \& Zinnecker 2004). Since we focus on brown dwarfs in Cha I, where there is no such hot star, we will not discuss this scenario further.

The ejection process might leave an observable imprint in the kinematics of members ejected from a cluster in comparison to that of non-ejected members. In order to test this scenario, Joergens \& Guenther (2001) carried out a precise kinematic analysis of brown dwarfs in Cha I and compared it to that of $\mathrm{T}$ Tauri stars in the same field based on mean radial velocities $(R V \mathrm{~s})$ measured from high-resolution spectra taken with the UV-Visual Echelle Spectrograph (UVES) at the Very Large Telescope (VLT). In this paper, we now present an improved analysis of this study based on additional $R V$ measurements with UVES of several of the targets in 2002 and 2004, based on a revised data analysis and on a cleaned and updated T Tauri star sample as comparison. Furthermore, the dispersion measured in terms of full width at half maximum (fwhm) in Joergens \& Guenther (2001) was misinterpreted in the literature as standard deviation. There is more than a factor of two difference between both quantities. In this paper, we also give the dispersion in terms of the standard deviation and then discuss the implications. We provide an empirical constraint for the kinematic properties of the studied group of very young brown dwarfs in Cha I and discuss the results in the context of current ideas about the formation of brown dwarfs and, in particular, the theoretical predictions of the embryo-ejection model.

It is noted that the UVES spectra were taken within the framework of an ongoing $R V$ survey for planetary and brown dwarf companions to young brown dwarfs and very low-mass stars in Cha I, which was published elsewhere (Joergens 2003, 2006).

The paper is organized as follows: Sect. 2 contains information about the UVES spectroscopy, data analysis, and the sample. In the next two sections, the kinematic study of brown dwarfs (Sect. 3) and of T Tauri stars in Cha I (Sect.4) is presented. Section 5 contains a discussion of the results and a comparison with theoretical predictions, and Sect. 6 conclusions and a summary.

\section{UVES spectroscopy, RV determination, and sample}

High-resolution spectra were taken for twelve brown dwarfs and (very) low-mass stars in Cha I between the years 2000 and 2004 with the echelle spectrograph UVES (Dekker et al. 2000) attached to the $8.2 \mathrm{~m}$ Kueyen telescope of the VLT operated by the European Southern Observatory at Paranal, Chile. Details of the data acquisition, analysis, and $R V$ determination are given in Joergens (2006). However, we point out here that the errors given in Table 2 of Joergens \& Guenther (2001) refer solely to relative errors. Additionally, an error of about $400 \mathrm{~m} \mathrm{~s}^{-1}$ due to the uncertainty in the zero offset of the template has to be taken into account for the absolute $R V$.

The targets of these observations are brown dwarfs and (very) low-mass stars with an age of a few million years situated in the center of the nearby $(\sim 160 \mathrm{pc})$ Cha I star-forming cloud (Comerón et al. 1999, 2000; Neuhäuser \& Comerón 1998, 1999). Membership in the Cha I cluster, and therefore the youth of the objects, is well established based on $\mathrm{H} \alpha$ emission, Lithium absorption, spectral types, and $R V \mathrm{~s}$ (references above; Joergens \& Guenther 2001; this work). The measured mean $R V \mathrm{~s}$ are listed in Table 1 for the nine M 6-M 8 type brown dwarfs and very low-mass stars Cha $\mathrm{H} \alpha 1-8$ and $\mathrm{Cha} \mathrm{H} \alpha 12$, while the mean $R V \mathrm{~s}$ for B34 (M 5), CHXR 74 (M4.5) and $\mathrm{Sz} 23$ (M 2.5) are included in Table 2 in the T Tauri star list.

Deviations of $R V$ s measured with UVES in this paper compared to $R V$ s presented in Joergens \& Guenther (2001) are attributed to a spectroscopic companion for Cha $\mathrm{H} \alpha 8$ (Joergens 2006) and to improved data reduction for the other objects. For the latter, these deviations lie in the range of $3-80 \mathrm{~m} \mathrm{~s}^{-1}$ with the exception of Cha $\mathrm{H} \alpha 7$ where the deviation was on the order of $3 \mathrm{~km} \mathrm{~s}^{-1}$, a situation that can be attributed to both the very low $\mathrm{S} / \mathrm{N}$ of the spectra of this object and a high sensitivity of the resulting $R V$ on the extraction algorithm for very low $\mathrm{S} / \mathrm{N}$ spectra.

\section{Kinematics of brown dwarfs in Cha I}

The determined mean $R V$ s for the nine M 6 to M 8-type brown dwarfs and very low-mass stars Cha $\mathrm{H} \alpha 1-8$ and $\mathrm{Cha} \mathrm{H} \alpha 12$ are given in Table 1 . They range between 14.5 and $17.1 \mathrm{~km} \mathrm{~s}^{-1}$ with an arithmetic mean of $15.71 \pm 0.31 \mathrm{~km} \mathrm{~s}^{-1}$. The $R V$ dispersion measured in terms of standard deviation of a population sample is $0.92 \mathrm{~km} \mathrm{~s}^{-1}$ with an uncertainty of $\pm 0.32 \mathrm{~km} \mathrm{~s}^{-1}$ derived from error propagation. The $R V$ dispersion measured in terms of fwhm is $2.15 \mathrm{~km} \mathrm{~s}^{-1}$. While the new mean $R V$ is larger than the value of $14.9 \mathrm{~km} \mathrm{~s}^{-1}$ given by Joergens \& Guenther (2001), their measurements of the total $R V$ range $\left(2.4 \mathrm{~km} \mathrm{~s}^{-1}\right)$ and of the fwhm dispersion $\left(2.0 \mathrm{~km} \mathrm{~s}^{-1}\right)$ differ only marginally from our results ${ }^{1}$.

The borderline between brown dwarfs and stars lies at about spectral type M7; i.e. the sample in Table 1 contains at least two objects, Cha H $\alpha 4$ and 5, which are most certainly of stellar nature. The substellar border defined by the hydrogenburning mass is a crucial dividing line with respect to further evolution of an object, but there is no obvious reason it should be of significance for the formation mechanism by

\footnotetext{
The fwhm is related to the standard deviation $\sigma$ of a Gaussian distribution by $f w h m=\sigma \sqrt{8 \ln 2}$; however, as pointed out by Sterzik \& Durisen (1998), the escape velocity distribution might be significantly non-Gaussian.
} 
Table 1. Mean $R V$ s for brown dwarfs and very low-mass stars in Cha I measured from UVES spectra. The values are the weighted mean of the individual time-resolved $R V$ s from Joergens (2006) and the error of the weighted mean plus $400 \mathrm{~m} \mathrm{~s}^{-1}$ for the error in the zero point of the velocity. For Cha $\mathrm{H} \alpha 8$, the $R V$ obtained in 2002 deviates significantly from the one obtained in 2000, hinting at a spectroscopic binary; therefore, the listed $R V$ of $\mathrm{Cha} \mathrm{H} \alpha 8$ is the arithmetic mean of the separately calculated 2000 and 2002 mean values.

\begin{tabular}{lcc}
\hline \hline Object & SpT & $\begin{array}{c}R V_{\text {UVES }} \\
{\left[\mathrm{km} \mathrm{s}^{-1}\right]}\end{array}$ \\
\hline Cha H $\alpha 1$ & M7.5 & $16.35 \pm 0.63$ \\
Cha H $\alpha 2$ & M 6.5 & $16.13 \pm 0.53$ \\
Cha H $\alpha 3$ & M 7 & $14.56 \pm 0.60$ \\
Cha H $\alpha 4$ & M 6 & $14.82 \pm 0.40$ \\
Cha H $\alpha 5$ & M 6 & $15.47 \pm 0.43$ \\
Cha H $\alpha 6$ & M7 & $16.37 \pm 0.68$ \\
Cha H $\alpha 7$ & M 8 & $17.09 \pm 0.98$ \\
Cha H $\alpha 8$ & M 6.5 & $16.08 \pm 1.62$ \\
Cha H $\alpha 12$ & M 7 & $14.50 \pm 0.96$ \\
\hline
\end{tabular}

which this object was produced. Thus, by whichever process brown dwarfs are formed, it is expected to work continuously into the regime of very low-mass stars. For an observational test to compare properties of brown dwarfs with those of stars, it is hence not a priori clear where to set the dividing line in mass of the two samples.

Therefore, we also consider the following samples: a) a subsample of Table 1 containing only brown dwarfs and brown dwarf candidates, i.e. all objects with spectral types M6.5 to M 8 (7 objects); as well as two larger samples that also include T Tauri stars from Table 2, namely, b) a sample of brown dwarfs and (very) low-mass stars with spectral types M4.5 to M 8 (11 objects); and c) a sample composed of all M-type (sub)stellar objects (M0-M 8, 17 objects). The mean $R V \mathrm{~s}$ of these samples (15.87 $\mathrm{km} \mathrm{s}^{-1}, 15.77 \mathrm{~km} \mathrm{~s}^{-1}, 15.36 \mathrm{~km} \mathrm{~s}^{-1}$ ) and their $R V$ dispersions (standard deviations: $0.97 \mathrm{~km} \mathrm{~s}^{-1}$, $0.83 \mathrm{~km} \mathrm{~s}^{-1}, 1.20 \mathrm{~km} \mathrm{~s}^{-1}$ ) do not differ significantly from the values derived for the brown dwarf sample (M 6-M 8) that was initially chosen. Also based on a Kolmogorov-Smirnov test, the $R V$ distributions of the samples a) to c) are consistent with the one of the M6-M 8 sample with a significance level of $\geq 99.98 \%$ for $a$ ) and $b$ ) and $98.50 \%$ for c).

\section{Kinematics of $\mathrm{T}$ Tauri stars in Cha I}

In order to compare the kinematics of the brown dwarfs in Cha I with higher-mass stellar objects in this cluster, we compiled all $\mathrm{T}$ Tauri stars that are confined to the same region for which $R V \mathrm{~s}$ have been measured with a precision of $2 \mathrm{~km} \mathrm{~s}^{-1}$ or better from the literature (Walter 1992; Dubath et al. 1996; Covino et al. 1997; Neuhäuser \& Comerón 1999), from Guenther et al. (in prep., see Joergens \& Guenther 2001), and from our own measurements based on UVES spectra for mid- to late M-type Cha I members. The result is a sample of $25 \mathrm{~T}$ Tauri stars (spectral types M 5-G2) as listed in Table 2. For nine of them, $R V$ measurements are available from more than one author and for two objects, CHX18N (Walter et al. 1992; Covino et al. 1997) and CHXR74 (Joergens 2006), $R V$ measurements at different epochs are significantly discrepant, which hints at longperiod spectroscopic companions. Table 2 gives the $R V \mathrm{~s}$ measured by the different authors as well as the derived mean $R V$ that was adopted for this study in the last column.

Compared to Joergens \& Guenther (2001), the T Tauri sample was revised by identifying double entries in their Table 2 , and by taking additional $R V \mathrm{~s}$ from the literature into account, by rejection of two foreground objects in the previous sample, by an improved data reduction for UVES-based $R V \mathrm{~s}$, as well as by additional UVES measurements. Details are given in the Appendix A.

We found that these $25 \mathrm{~T}$ Tauri stars have an arithmetic mean $R V$ of $14.73 \pm 0.25 \mathrm{~km} \mathrm{~s}^{-1}$, and an $R V$ dispersion in terms of a standard deviation of $1.26 \pm 0.31 \mathrm{~km} \mathrm{~s}^{-1}$ and in terms of an fwhm of $2.96 \mathrm{~km} \mathrm{~s}^{-1}$. Compared to the values given by Joergens \& Guenther (2001), namely a mean $R V$ of $14.9 \mathrm{~km} \mathrm{~s}^{-1}$, a standard deviation of $1.5 \mathrm{~km} \mathrm{~s}^{-1}$, and an fwhm of $3.6 \mathrm{~km} \mathrm{~s}^{-1}$, the new values are slightly lower. Interestingly, the difference in the dispersion can be solely attributed to the previously unresolved binarity of CHX18N, and the difference in the mean $R V$ can be partly attributed to this fact (cf. Appendix A).

As for the brown dwarf case, we also calculated the kinematics for subsamples in order to account for the possibility that, if brown dwarfs are formed by a different mechanism than stars, (very) low-mass stars might form in a brown dwarf like manner rather than a star like. No significant differences were found between the original $\mathrm{T}$ Tauri sample and a sample of only those stars with a larger mass than about $0.2 M_{\odot}$ (23 stars, M3.25-G2, mean $R V$ : $14.61 \mathrm{~km} \mathrm{~s}^{-1}, R V$ standard deviation: $1.24 \mathrm{~km} \mathrm{~s}^{-1}$ ) and a sample of only $\mathrm{K}$ and $\mathrm{G}$ type $\mathrm{T}$ Tauri stars (17 stars, K8-G2, mean $R V: 14.62 \mathrm{~km} \mathrm{~s}^{-1}, R V$ standard deviation: $1.21 \mathrm{~km} \mathrm{~s}^{-1}$ ). A Kolmogorov-Smirnov test also showed that the $R V$ distributions of the two subsamples are consistent with the one of the original $\mathrm{T}$ Tauri sample with significance levels above $99.52 \%$.

A kinematic difference between single and binary/multiple stars was suggested by Sterzik \& Durisen (2003) and Delgado-Donate et al. (2003) in the sense that the velocities of multiples are less dispersed. Therefore, we investigate the kinematics of the $\mathrm{T}$ Tauri stars in respect of their multiplicity status. A significant fraction of the $\mathrm{T}$ Tauri stars in our sample has been resolved into visual binaries ( $\mathrm{Sz}$ 19, Sz 20, Sz 41, F 34, CV Cha, SX Cha, VW Cha, CHX 22) by Reipurth \& Zinnecker (1993), Brandner et al. (1996), Brandner \& Zinnecker (1997), and Ghez et al. (1997). Furthermore, as mentioned above, there are indications of spectroscopic companions around CHX18N (Walter 1992; Covino et al. 1997) and CHXR 74 (Joergens 2006). Thus, among the sample of $25 \mathrm{~T}$ Tauri stars, at least about 10 are in binary or multiple systems. We calculated a mean $R V$ of $14.68 \mathrm{~km} \mathrm{~s}^{-1}$ and an $R V$ dispersion (standard deviation) of $1.02 \mathrm{~km} \mathrm{~s}^{-1}$ for the sample of the $10 \mathrm{~T}$ Tauri stars in binary or multiple systems and a mean $R V$ of $14.76 \mathrm{~km} \mathrm{~s}^{-1}$ and a $R V$ dispersion (standard deviation) of $1.42 \mathrm{~km} \mathrm{~s}^{-1}$ for the remaining stars of the sample, which might, for the most part, be single. Thus, the $R V \mathrm{~s}$ of the sample of "single" $\mathrm{T}$ Tauri stars in 
Table 2. List of T Tauri stars in Cha I with known $R V \mathrm{~s}$ ordered by spectral type. All T Tauri stars in Cha I were compiled with $R V \mathrm{~s}$ known to a precision of $2 \mathrm{~km} \mathrm{~s}^{-1}$ or better from our UVES observations and from the literature. $R V_{\mathrm{UVES}}$ values are measured from our UVES spectra and are weighted means based on several measurements, and the error takes into account $400 \mathrm{~m} \mathrm{~s}^{-1}$ for the error in the zero point of the velocity. The last column gives the $R V$ adopted for the kinematic study. For objects with more than one available $R V$ measurement, usually the weighted average and the error of the weighted mean are given. An exception of this was made for CHX18N and CHXR 74 , for which $R V \mathrm{~s}$ at different epochs are significantly deviant; and, therefore, the arithmetic mean of the different epoch $R V \mathrm{~s}$ is given. The listed names are those used by the authors of the $R V$ data. In addition, for each object we give the name used by the most recent compilation of Cha I members (Luhman 2004), e.g. "T5" stands for the Simbad name "Ass Cha T 2-5". Errors for CS Cha, VZ Cha, WY Cha are not given by the author and are rough estimates based on an average error for the remaining $R V \mathrm{~s}$ determined by the same author. References are W92: Walter (1992); D96: Dubath et al. (1996); C97: Covino et al. (1997); N99: Neuhäuser \& Comerón (1999); G: Guenther et al., in prep.; UVES: Joergens \& Guenther (2001), Joergens (2003), Joergens (2006), this work.

\begin{tabular}{|c|c|c|c|c|c|c|c|c|c|}
\hline Object & Other names & SpT & $\begin{array}{c}R V_{\mathrm{W} 92} \\
{\left[\mathrm{~km} \mathrm{~s}^{-1}\right]}\end{array}$ & $\begin{array}{c}R V_{\mathrm{D} 96} \\
{\left[\mathrm{~km} \mathrm{~s}^{-1}\right]}\end{array}$ & $\begin{array}{c}R V_{\mathrm{C} 97} \\
{\left[\mathrm{~km} \mathrm{~s}^{-1}\right]}\end{array}$ & $\begin{array}{c}R V_{\mathrm{N} 99} \\
{\left[\mathrm{~km} \mathrm{~s}^{-1}\right]}\end{array}$ & $\begin{array}{c}R V_{\mathrm{G}} \\
{\left[\mathrm{km} \mathrm{s}^{-1}\right]}\end{array}$ & $\begin{array}{c}R V_{\mathrm{UVES}} \\
{\left[\mathrm{km} \mathrm{s}^{-1}\right]}\end{array}$ & $\begin{array}{c}R V_{\text {mean }} \\
{\left[\mathrm{km} \mathrm{s}^{-1}\right]}\end{array}$ \\
\hline Sz 19 & DICha, CHX9, T26 & G2 & $14 \pm 2$ & $13.5 \pm 0.6$ & & & & & $13.5 \pm 0.1$ \\
\hline CHX 7 & $\mathrm{~T} 21$ & G5 & $17 \pm 2$ & & & & & & $17 \pm 2$ \\
\hline CHX 22 & T54 & G8 & $14 \pm 2$ & & & & & & $14 \pm 2$ \\
\hline CV Cha & $\mathrm{Sz} 42, \mathrm{~T} 52$ & G9 & & $15.1 \pm 0.3$ & & & $15.6 \pm 0.9$ & & $15.2 \pm 0.2$ \\
\hline Sz 6 & CR Cha, T8 & $\mathrm{K} 2$ & & $14.9 \pm 0.8$ & & & & & $14.9 \pm 0.8$ \\
\hline F 34 & WKK F34, CHXR47 & K3 & & & $14.0 \pm 2.0$ & & & & $14.0 \pm 2.0$ \\
\hline $\mathrm{Sz} 41$ & T51,RXJ1112.7-7637 & $\mathrm{K} 3.5$ & & $13.9 \pm 0.4$ & $16 \pm 2$ & & & & $14.0 \pm 0.4$ \\
\hline CHX20E & CHXR55 & $\mathrm{K} 4.5$ & $13 \pm 2$ & & & & & & $13 \pm 2$ \\
\hline CT Cha & Sz $11, \mathrm{~T} 14$ & K5 & & $15.1 \pm 0.5$ & & & $15.5 \pm 1.4$ & & $15.1 \pm 0.1$ \\
\hline CHX18N & RXJ1111.7-7620 & K6 & $13 \pm 2$ & & $19.0 \pm 2.0$ & & & & $16.0 \pm 3.0$ \\
\hline CHX 10a & CHX 10 , CHXR 28 & K6 & $16 \pm 2$ & & & & & & $16 \pm 2$ \\
\hline VZ Cha & $\mathrm{T} 40$ & K6 & & & & & $14.7 \pm 0.8$ & & $14.7 \pm 0.8$ \\
\hline CS Cha & Sz $9, \mathrm{~T} 11$ & K6 & & $14.7 \pm 0.3$ & & & $14.9 \pm 0.8$ & & $14.7 \pm 0.1$ \\
\hline CHXR 37 & RXJ1109.4-7627 & K7 & & & $13.1 \pm 2.0$ & & & & $13.1 \pm 2.0$ \\
\hline TW Cha & $\mathrm{T} 7$ & K8 & & & & & $15.7 \pm 1.2$ & & $15.7 \pm 1.2$ \\
\hline VW Cha & $\mathrm{T} 31$ & K8 & & & & & $15.1 \pm 0.1$ & & $15.1 \pm 0.1$ \\
\hline WY Cha & Sz 36, T46 & K8 & & $12.9 \pm 0.9$ & & & $12.1 \pm 0.8$ & & $12.5 \pm 0.4$ \\
\hline SX Cha & $\mathrm{T} 3$ & M 0.5 & & & & & $13.4 \pm 0.9$ & & $13.4 \pm 0.9$ \\
\hline SY Cha & $\mathrm{T} 4$ & M 0.5 & & & & & $12.7 \pm 0.1$ & & $12.7 \pm 0.1$ \\
\hline CHX 21a & CHX 21, CHXR 54 & M 1 & $14 \pm 2$ & & & & & & $14 \pm 2$ \\
\hline Sz 20 & VV Cha, T27 & M 1 & & $15.4 \pm 1.3$ & & & & & $15.4 \pm 1.3$ \\
\hline Sz 23 & $\mathrm{~T} 30$ & M 2.5 & & & & & & $15.57 \pm 0.55$ & $15.57 \pm 0.55$ \\
\hline $\mathrm{Sz} 4$ & $\mathrm{~T} 5$ & M3.25 & & $16.5 \pm 1.3$ & & & & & $16.5 \pm 1.3$ \\
\hline \multirow[t]{2}{*}{ CHXR 74} & & M 4.5 & & & & $16.5 \pm 1.0$ & & $14.58 \pm 0.62$ & \\
\hline & & & & & & & & $17.42 \pm 0.44$ & $16.2 \pm 0.8$ \\
\hline B 34 & CHXR 76 & M 5 & & & & $17.6 \pm 1.7$ & & $15.75 \pm 0.42$ & $15.9 \pm 0.4$ \\
\hline
\end{tabular}

Cha I are slightly more dispersed than the T Tauri binary stars, which are mainly visual and, hence, wide systems, as further discussed in the next section.

\section{Discussion}

\subsection{Observational results for Chal}

The mean $R V$ of the M 6-M 8 type brown dwarfs and very lowmass stars $\left(15.7 \pm 0.3 \mathrm{~km} \mathrm{~s}^{-1}\right)$ is consistent with that of the surrounding molecular gas (15.4 $\mathrm{km} \mathrm{s}^{-1}$, Mizuno et al. 1999) in agreement with membership of this substellar population in the
Cha I star-forming cloud. In Fig. 1 the $R V \mathrm{~s}$ determined for the brown dwarfs in Cha I are compared to those of T Tauri stars in the same region in the form of a histogram. It can be seen that the $R V \mathrm{~s}$ of the brown dwarfs are on average larger, by 1.8 times the errors, than those for the T Tauri stars $\left(14.7 \pm 0.3 \mathrm{~km} \mathrm{~s}^{-1}\right)$. The dispersion of the $R V \mathrm{~s}$ of the brown dwarfs measured in terms of standard deviation $\left(0.9 \pm 0.3 \mathrm{~km} \mathrm{~s}^{-1}\right)$ is slightly lower than that of the $\mathrm{T}$ Tauri stars $\left(1.3 \pm 0.3 \mathrm{~km} \mathrm{~s}^{-1}\right)$, and both are (slightly) larger than that of the surrounding molecular gas (the fwhm of $0.9 \mathrm{~km} \mathrm{~s}^{-1}$ given by Mizuno et al. (1999) for the average cloud core translates to a standard deviation of $0.4 \mathrm{~km} \mathrm{~s}^{-1}$ ). While the differences in the dispersion values lie well within 


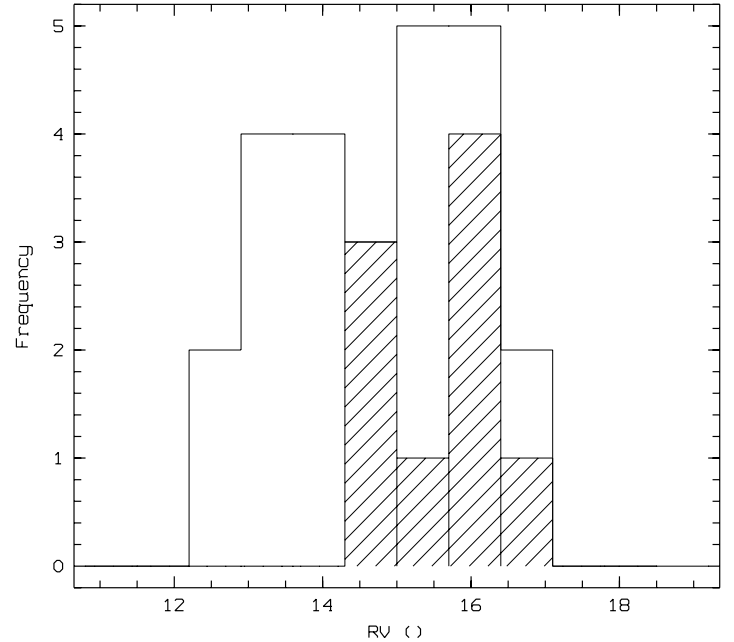

Fig. 1. Histogram of mean $R V \mathrm{~s}$ in $\left[\mathrm{km} \mathrm{s}^{-1}\right]$ of nine brown dwarfs and very low-mass stars in Cha I ( $\leq 0.1 M_{\odot}, \mathrm{M} 6-\mathrm{M} 8$, hashed) and of 25 T Tauri stars (M 5-G2) in the same field.

the error range, it is unlikely from the data that the $R V \mathrm{~s}$ of the brown dwarfs are significantly more dispersed than that of the $\mathrm{T}$ Tauri stars.

Since the velocity distribution arising from the dynamical decay of $N$-body clusters might be significantly non-Gaussian (Sterzik \& Durisen 1998), the determined $R V \mathrm{~s}$ of the brown dwarfs and T Tauri stars in Cha I are also analyzed in the form of cumulative distributions. In Fig. 2, the fraction of objects with a relative $R V$ smaller or equal to a given relative $R V$ is plotted. The relative $R V$ is given by the absolute value of the deviation from the mean of the whole group. Since the measured mean $R V \mathrm{~s}$ for the brown dwarfs and $\mathrm{T}$ Tauri stars deviate slightly but significantly, these two different mean $R V \mathrm{~s}$ are used. It can be seen that the cumulative $R V$ distributions for brown dwarfs and $\mathrm{T}$ Tauri stars in Cha I diverge for higher velocities. While the cumulative $R V$ distribution of the brown dwarfs displays a linear and steeper increase, with none of them having an $R V$ deviating from the mean by more than $1.4 \mathrm{~km} \mathrm{~s}^{-1}$, the T Tauri stars show a tail of higher velocities: $28 \%$ have an $R V$ deviating from the mean by more than $1.4 \mathrm{~km} \mathrm{~s}^{-1}$. An examination of the $R V$ distributions of the studied brown dwarf and $\mathrm{T}$ Tauri star sample in Cha I based on Kolmogorov-Smirnov statistics also indicates a significant difference. The significance level for the consistency of the distributions of the absolute $R V \mathrm{~s}$ is only $11.70 \%$, while it is $74.30 \%$ for the relative $R V$ s considered in the same manner as for Fig. 2.

That the improved value for the $R V$ dispersion in terms of standard deviation of the T Tauri stars is slightly smaller than the value found by Joergens \& Guenther (2001) can be attributed solely to the unresolved binarity of one of the T Tauri stars in the sample. This demonstrates that the orbital motion of unresolved spectroscopic binaries are a source of error. An additional possible error source is $R V$ variability induced by surface spots. Given the recent indications that $R V$ noise caused by surface activity is very small for brown dwarfs in Cha I (Joergens 2006), the slightly higher value for the $R V$ dispersion of $\mathrm{T}$ Tauri stars compared to brown dwarfs might be

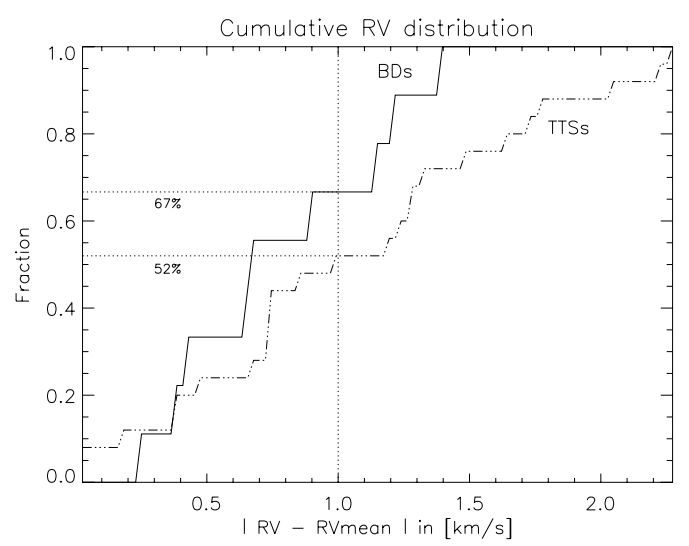

Fig. 2. Cumulative $R V$ distribution for brown dwarfs (solid line) and T Tauri stars (dash-dotted line). The samples are the same as for Fig. 1. Plotted is the fraction of objects with a relative $R V$ smaller or equal to a given relative $R V$. It can be seen that the $R V$ of none of the brown dwarfs deviates from the mean $R V$ of the brown dwarfs by more than $1.4 \mathrm{~km} \mathrm{~s}^{-1}$ and that $67 \%$ of them have $R V$ deviations smaller or equal to $0.9 \mathrm{~km} \mathrm{~s}^{-1}$ compared to $52 \%$ for the T Tauri stars with $R V$ deviations smaller or equal to $1.0 \mathrm{~km} \mathrm{~s}^{-1}$.

attributed to such systematic errors being more pronounced in the stellar than in the substellar regime. While the $R V \mathrm{~s}$ of the $\mathrm{T}$ Tauri stars have to a large degree been collected from the literature and obtained with a variety of instruments and wavelength resolutions, there is no hint that this introduces a systematic error. First, for seven out of the nine T Tauri stars for which $R V \mathrm{~s}$ have been measured by more than one author, consistent $R V \mathrm{~s}$ were found despite different instrumentation. Secondly, $R V$ dispersions and mean values of the T Tauri subsamples in Table 2 sorted by author, i.e. obtained in a uniform way, agree very well with the kinematic properties derived here for the total sample of compiled T Tauri stars. For example, the $\mathrm{T}$ Tauri stars observed by Guenther et al. have an $R V$ dispersion of $1.3 \mathrm{~km} \mathrm{~s}^{-1}$ and a mean value of $14.4 \mathrm{~km} \mathrm{~s}^{-1}$; the ones observed by Dubath et al. (1996) have $1.1 \mathrm{~km} \mathrm{~s}^{-1}$ and $14.7 \mathrm{~km} \mathrm{~s}^{-1}$; and the ones observed by Walter (1992) have $1.5 \mathrm{~km} \mathrm{~s}^{-1}$ and $14.4 \mathrm{~km} \mathrm{~s}^{-1}$.

An investigation of the $R V$ s of the T Tauri stars in Cha I taking their multiplicity status into account showed that the sample of 10 stars for which indications of visual or spectroscopic binaries were detected has less dispersed $R V \mathrm{~s}$ (standard deviation $1.02 \pm 0.57 \mathrm{~km} \mathrm{~s}^{-1}$ ) compared to the remaining 15 "single" stars of the sample $\left(1.42 \pm 0.41 \mathrm{~km} \mathrm{~s}^{-1}\right)$, while these values are still consistent within the errors. In Fig. 3, cumulative $R V$ distributions for T Tauri binaries and "singles" are plotted. A trend of less dispersed velocities for the binaries can be seen, with $60 \%$ of the T Tauri "singles" having $v \leq 1.5 \mathrm{~km} \mathrm{~s}^{-1}$ but $100 \%$ of $\mathrm{T}$ Tauri binaries having $v<1.5 \mathrm{~km} \mathrm{~s}^{-1}$. A KolmogorovSmirnov test also indicates that the $R V$ distributions of these samples might be different (71.59\%). A similar study for the brown dwarfs in Cha I is not possible yet since among the brown dwarf sample, so far only one has shown any indication of binarity (Cha $\mathrm{H} \alpha$ 8, Joergens 2006). The fact that this object has one of the smallest deviations from the mean of the whole group, namely $0.4 \mathrm{~km} \mathrm{~s}^{-1}$, is in line with observations of the 


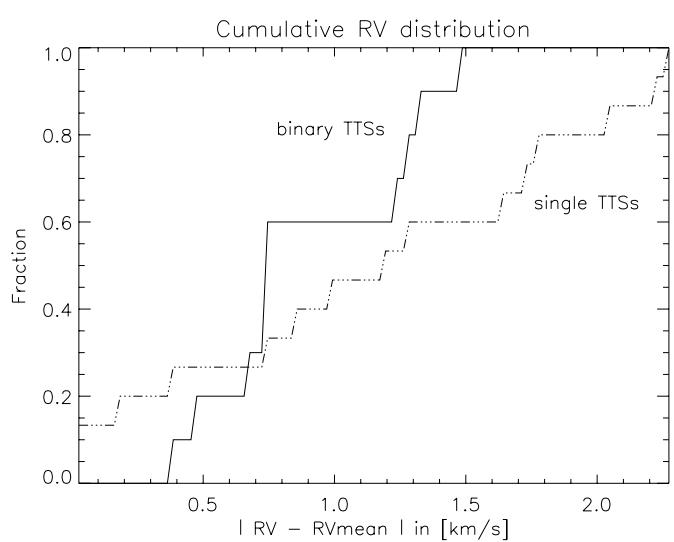

Fig. 3. Cumulative $R V$ distributions for T Tauri "singles" (dash-dotted line) and binaries / multiples (solid line). Note the steeper rise of the binary subsample indicating an absence of a high velocity tail compared to those $\mathrm{T}$ Tauri stars regarded as single.

T Tauri binaries. However, several of the (sub)stellar objects regarded as "single" may still be resolved into multiple systems in the future. Since nine out of these ten T Tauri binaries are visual, hence, wide binaries, we can assume that predominantly spectroscopic or, at least, close systems have still not been resolved. Thus, this kinematic difference might translate into a kinematic difference between the group of wide binaries and the group of single stars and close binaries.

\subsection{Comparing Cha I and Taurus observations}

Comparing our kinematic study in Cha I with $R V$ measurements for the Taurus star-forming region shows that, in agreement with our finding for Cha I, the $R V$ dispersion for brown dwarfs in Taurus does not seem to deviate significantly from that of $\mathrm{T}$ Tauri stars in this cloud. The $R V$ dispersion measured in terms of the standard deviation of six brown dwarfs and very low-mass stars in Taurus (M 6-M 7.5) is $1.9 \mathrm{~km} \mathrm{~s}^{-1}$ (White \& Basri 2003) and that for $38 \mathrm{~T}$ Tauri stars in the same cloud is $2.1 \mathrm{~km} \mathrm{~s}^{-1}$ (Hartmann et al. 1986).

There are still two differences compared to the situation in Cha I. In Fig. 4, the cumulative $R V$ distributions are plotted for both Taurus samples based on the $R V$ s published by the authors. Both follow the same distribution in this diagram, in contrast to the case for Cha I (Fig. 2) where the cumulative $R V$ distributions for brown dwarfs and stars diverge for higher velocities.

Secondly, the $R V$ dispersions for Taurus brown dwarfs and stars are significantly higher than the ones for Cha I brown dwarfs $\left(0.9 \mathrm{~km} \mathrm{~s}^{-1}\right)$ and stars $\left(1.3 \mathrm{~km} \mathrm{~s}^{-1}\right)$. We measured a global $R V$ dispersion of $1.24 \pm 0.24 \mathrm{~km} \mathrm{~s}^{-1}$ for all Cha I brown dwarfs and stars in Tables 1 and 2. For comparison, the velocity dispersion of the molecular gas in the Cha I cloud cores is on average $0.4 \mathrm{~km} \mathrm{~s}^{-1}$ in terms of the standard deviation (Mizuno et al. 1999). For Taurus, we found ${ }^{2}$ a global $R V$ dispersion of $2.04 \pm 0.30 \mathrm{~km} \mathrm{~s}^{-1}$ for the sample of brown dwarfs and

\footnotetext{
${ }^{2}$ For the calculation of the error of the dispersion, the individual errors of the $R V$ values are necessary, which are not always given by Hartmann et al. (1986). In the cases of absent individual errors, we used an average error derived from the given individual errors.
}

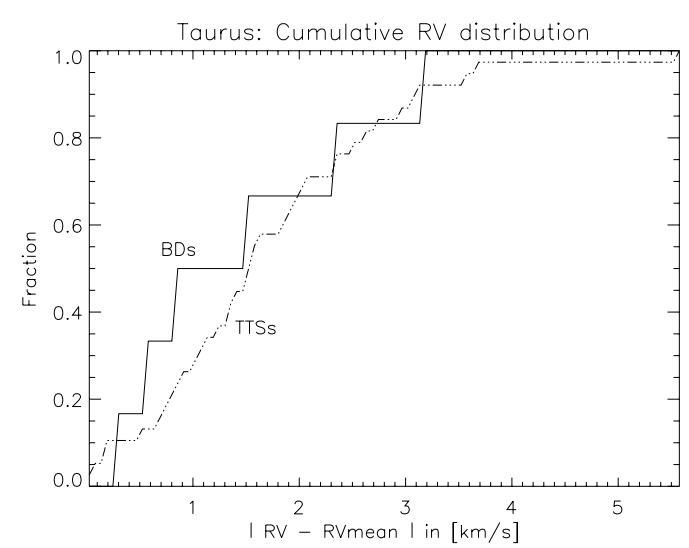

Fig. 4. For comparison, cumulative $R V$ distributions for members of Taurus: brown dwarfs, very low-mass stars (M 6 and later, six objects, White \& Basri 2003, solid line), and higher mass T Tauri stars (38 objects, Hartmann et al. 1986, dash-dotted line).

(very) low-mass stars in White \& Basri (2003) combined with the sample of T Tauri stars in Hartmann et al. (1986). Again for comparison, the velocity dispersion of the molecular gas in the Taurus cloud cores is on average $0.3 \mathrm{~km} \mathrm{~s}^{-1}$ in terms of standard deviation (Onishi et al. 1996). We conclude that the $R V$ dispersion for Cha I and Taurus (sub)stellar members deviate significantly. The $R V$ dispersion for Taurus is about a factor of two higher than for Cha I, while Taurus has a much lower stellar density and star-formation efficiency (Oasa et al. 1999; Tachihara et al. 2002). Thus, a fundamental increase in velocity dispersion with stellar density of the star-forming region, as suggested by Bate \& Bonnell (2005), is not established observationally (see also Sect. 5.3.3).

\subsection{Comparison with theoretical models}

\subsubsection{Overview of models}

Enormous theoretical efforts have been undertaken in recent years to model the formation of brown dwarfs by the embryoejection mechanism and to simulate the dynamical evolution in star-forming regions. Hydrodynamical calculations of the collapse of a $50 M_{\odot}$ cloud (Bate et al. 2002, 2003; Bate \& Bonnell 2005) have demonstrated that brown dwarfs are formed in these models as stellar seeds that are ejected early. While predictions of (sub)stellar parameters by these models are based on small numbers, this can be overcome by the combination of collapse calculations with $\mathrm{N}$-body simulations of further dynamical evolution (Delgado-Donate et al. 2003, 2004). However, the predicted properties of current hydrodynamical models have been questioned because of the lack of feedback processes (Kroupa $\&$ Bouvier 2003a).

On the other hand, simulations of the dynamical evolution of small $N$-body clusters (Sterzik \& Durisen 2003) made statistically robust predictions of the properties of very young brown dwarfs and stars possible. There have been recent efforts to incorporate further details of the star-formation process, in particular ongoing accretion during the dynamical interactions (Umbreit et al. 2005). However, the current $N$-body simulations 
do not consider the gravitational potential of the cluster, which might significantly influence, e.g., the predicted velocities.

An approach to estimating the gravitational potential of existing star-forming regions has been suggested by Kroupa $\&$ Bouvier (2003b) for Taurus-Auriga and the Orion Nebula Cluster (ONC). However, the assumed cloud core properties are not straightforward to understand. While the cluster mass (stars and gas) of $9000 M_{\odot}$ adopted by the authors for the ONC is plausible for the embedded gas of the whole ONC at birth time (Wilson et al. 2005), the value of $50 M_{\odot}$ for Taurus-Auriga corresponds to about twice the mass of one $\mathrm{C} 18 \mathrm{O}$ cloud core in Taurus (Onishi et al. 1996). Furthermore, since Cha I cloud cores have a similar average mass and radius (Mizuno et al. 1999) to Taurus, we would find a similar estimate for the gravitational potential when following along these lines, while there is a large difference in stellar density and star-formation efficiency between these regions (Oasa et al. 1999; Tachihara et al. 2002).

\subsubsection{RVs and 3D velocities in Cha I}

When considering dynamical evolutions, the transformation from $R V$ s to $3 \mathrm{D}$ velocities is not straightforward, but instead depends on details of the gravitational potential of the cluster and on the number of objects with very small velocities, i.e. the details of the simulations, as explained in the following. The brown dwarfs studied in Cha I are situated in a relatively densely populated region of Cha I at the periphery of one of its six cloud cores, the so-called "YSO condensation B" (Mizuno et al. 1999). They occupy a field of less than $0.2 \times 0.2 \mathrm{deg}$ at a distance of $160 \mathrm{pc}$. Having an age of about $2 \mathrm{Myr}$ (Comerón et al. 2000), the brown dwarfs born within this field and ejected during their formation in directions with a significant fraction perpendicular to the line-of-sight, would have already vanished for velocities of $0.4 \mathrm{~km} \mathrm{~s}^{-1}$ or larger (Joergens et al. 2003a). With a velocity of $\sim 0.8 \mathrm{~km} \mathrm{~s}^{-1}$, an object can even cross the whole extent of the YSO condensation B $(\sim 0.2 \times 0.5 \mathrm{deg})$. In the case of a significant fraction of brown dwarfs leaving the survey area, the remaining observable objects will be of two sorts: those with very small velocities (too small to travel the extent of the region $\left(<0.4 \mathrm{~km} \mathrm{~s}^{-1}\right)$ and/or too small to overcome the binding energy of the cluster) and those with larger velocities but moving predominantly in a radial direction. Due to this selection of fast-moving objects predominantly in a radial direction, the observed $R V$ dispersion of such a group in such a limited survey area would be larger than the calculated $1 \mathrm{D}$ velocities that consider all objects regardless of the distance to the birth place. On the other hand, the observed $R V$ dispersion would still be smaller than the calculated $3 \mathrm{D}$ velocities given the majority of bound objects with a small velocity component.

\subsubsection{Comparison with hydrodynamical calculations}

The brown dwarfs and stars formed in the hydrodynamical model by Bate et al. (2003) share the same kinematic properties. For a stellar density of $1.8 \times 10^{3} \mathrm{stars} / \mathrm{pc}^{3}$, they find an rms velocity dispersion of $2.1 \mathrm{~km} \mathrm{~s}^{-1}$ in $3 \mathrm{D}$. Identical calculations for a denser star-forming region $(2.6 \times$ $10^{4} \mathrm{stars} / \mathrm{pc}^{3}$, Bate \& Bonnell 2005) yield an rms velocity dispersion of $4.3 \mathrm{~km} \mathrm{~s}^{-1}$. The combined hydrodynamic / $N$-body model by Delgado-Donate et al. (2004) also predict no, or at most slight kinematic differences between ejected and non-ejected members of a cluster. The 3D velocity dispersion of the produced objects is $2-3 \mathrm{~km} \mathrm{~s}^{-1}$ (Delgado-Donate, Pers. Comm.) for modeled stellar densities of $2.5-3.6 \times 10^{4} \mathrm{stars} / \mathrm{pc}^{3}$ (Delgado-Donate et al. 2004).

The theoretical finding that there is no kinematic difference between stars and brown dwarfs is consistent with our measurement of no significant difference between the $R V$ dispersion of brown dwarfs $\left(0.9 \pm 0.3 \mathrm{~km} \mathrm{~s}^{-1}\right)$ and stars $\left(1.3 \pm 0.3 \mathrm{~km} \mathrm{~s}^{-1}\right)$ in Cha I. However, the $R V$ dispersions observed in Cha I for both brown dwarfs and stars are much smaller than predicted by the models. Since the stellar densities in these calculations (on the order of $10^{3}$ to $10^{4} \mathrm{stars} / \mathrm{pc}^{3}$ ) are much higher than in the Cha I cloud (on the order of $10^{2}$ stars $/ \mathrm{pc}^{3}$, Oasa et al. 1999), one could argue that extrapolation towards smaller densities might explain the discrepancy. An extrapolation of the trend seen in the models by Bate et al. (2003) and Bate \& Bonnell (2005) towards the stellar densities in Cha I, for instance, would yield a velocity dispersion of about $1 \mathrm{~km} \mathrm{~s}^{-1}$ that is consistent with the $R V$ dispersion measured for Cha I. However, the same extrapolation yields a value that is highly inconsistent with the observations for Taurus that has a much smaller observed stellar density than Cha I (see Sect. 5.2). Furthermore, there are apparently inconsistencies between different theoretical models: the model from Delgado-Donate et al. (2004) produces similar stellar densities as calculation 2 of Bate \& Bonnell (2005), but at the same time both predict very different velocity dispersions of 2-3 $\mathrm{km} \mathrm{s}^{-1}$ (Delgado-Donate, Pers. Comm.) and $4.3 \mathrm{~km} \mathrm{~s}^{-1}$ (Bate \& Bonnell 2005). We therefore conclude that the dependence of the velocity dispersion on the stellar densities has not yet been established and an extrapolation is not advisable.

\subsubsection{Comparison with $N$-body simulations}

The decay models of Sterzik \& Durisen (2003) predict that $25 \%$ of the brown dwarf singles have a velocity that is smaller than $1 \mathrm{~km} \mathrm{~s}^{-1}$. This is a smaller percentage than found by our observations that $67 \%$ of the brown dwarfs in Cha I have $R V \mathrm{~s}$ smaller than $1 \mathrm{~km} \mathrm{~s}^{-1}$. Furthermore, Sterzik \& Durisen (2003) find a high-velocity tail with $40 \%$ single brown dwarfs having $v>1.4 \mathrm{~km} \mathrm{~s}^{-1}$ and $10 \%$ having $v>5 \mathrm{~km} \mathrm{~s}^{-1}$. This is also not seen in our data, where none has an $R V$ deviating by the mean $R V$ of the group by more than $1.4 \mathrm{~km} \mathrm{~s}^{-1}$. Admittedly, the relatively small size of our brown dwarf sample does not exclude the possibility that we have missed the $40 \%$ objects moving faster than $1.4 \mathrm{~km} \mathrm{~s}^{-1}$.

In the formation phase where dynamical interactions become important, gas accretion might still be a significant factor. $N$-body simulations by Umbreit et al. (2005) find higher ejection velocities for models taking ongoing accretion during the dynamical encounters into account. They predict that between $60 \%$ and almost $80 \%$ of single brown dwarfs have velocities larger than $1 \mathrm{~km} \mathrm{~s}^{-1}$ depending on the accretion rates. 
That is much larger than found by our observations, where only about $30 \%$ of the brown dwarfs have velocities $>1 \mathrm{~km} \mathrm{~s}^{-1}$.

Sterzik \& Durisen (2003) furthermore find different kinematics for singles and binaries: $90 \%$ of their stellar binaries have velocities smaller than $1 \mathrm{~km} \mathrm{~s}^{-1}$, while only $50 \%$ of stellar singles can be found in that velocity range. This agrees with our tentative finding that the subgroup of binaries among the studied sample of $\mathrm{T}$ Tauri stars in Cha I has a lower $R V$ dispersion $\left(1.0 \mathrm{~km} \mathrm{~s}^{-1}\right)$ and no high-velocity tail (Fig. 3) compared to the remaining "single" stars $\left(1.42 \mathrm{~km} \mathrm{~s}^{-1}\right)$. However, the T Tauri "single" star sample might be contaminated by unresolved binaries.

\section{Conclusions and summary}

In order to pave the way to an understanding of the still unknown origins of brown dwarfs, we explored the kinematic properties of extremely young brown dwarfs in the starforming cloud Cha I based on precise $R V \mathrm{~s}$ measured from highresolution UVES / VLT spectra. This kinematic study is an improved version of the one by Joergens \& Guenther (2001), which provided the first observational constraints for the velocity distribution of a group of very young brown dwarfs.

We found that nine brown dwarfs and very low-mass stars (M 6-M 8, $M \lesssim 0.1 M_{\odot}$ ) in Cha I kinematically form a very homogeneous group. They have very similar absolute $R V \mathrm{~s}$ with a mean value of $15.7 \mathrm{~km} \mathrm{~s}^{-1}$, an $R V$ dispersion in terms of standard deviation of $0.9 \mathrm{~km} \mathrm{~s}^{-1}$, and a total covered $R V$ range of $2.6 \mathrm{~km} \mathrm{~s}^{-1}$.

We conducted a comparison of the kinematic properties of these brown dwarfs with those of $25 \mathrm{~T}$ Tauri stars confined to the same field based on our UVES measurements, as well as on $R V$ s from the literature. For the $\mathrm{T}$ Tauri stars, we determined a mean $R V$ of $14.7 \mathrm{~km} \mathrm{~s}^{-1}$, a $R V$ dispersion of $1.3 \mathrm{~km} \mathrm{~s}^{-1}$, and a total range of $4.5 \mathrm{~km} \mathrm{~s}^{-1}$.

The mean $R V \mathrm{~s}$ of the brown dwarfs are larger than that of the T Tauri stars by less than two times the errors; however, both values are consistent with the velocity of the molecular gas of the surroundings. The $R V$ dispersion measured for the brown dwarfs is slightly smaller than the one for the T Tauri stars, but this difference lies within the errors. We found that the cumulative $R V$ distributions for the brown dwarfs and for the T Tauri stars diverge for $R V \mathrm{~s}$ higher than about $1 \mathrm{~km} \mathrm{~s}^{-1}$, with the brown dwarfs displaying no tail with high velocities in contrast to the T Tauri stars. This could be an intrinsic feature or might be attributed to more pronounced systematic $R V$ errors in the stellar than in the substellar mass domain.

The finding of consistent $R V$ dispersions for brown dwarfs and stars in ChaI (Joergens \& Guenther 2001; this paper) is also seen in $R V$ data for brown dwarfs and stars in the Taurus star-forming region (Hartmann et al. 1986; White \& Basri 2003). We calculated global $R V$ dispersions for all brown dwarfs and stars in Cha I $\left(1.24 \mathrm{~km} \mathrm{~s}^{-1}\right)$ and Taurus $\left(2.0 \mathrm{~km} \mathrm{~s}^{-1}\right)$ and found that the value for Taurus is significantly higher than the one for Cha I by about a factor of two. Given that the stellar density of Taurus is much smaller than of Cha I (Oasa et al. 1999), we conclude that a fundamental increase of velocity dispersion with stellar density of the star-forming region as suggested by Bate \& Bonnell (2005) is not established observationally.

We compared the kinematic study in Cha I with theoretical hydrodynamical or $\mathrm{N}$-body calculations of the embryoejection scenario for the formation of brown dwarfs. That there is no significant difference between the $R V$ dispersion of brown dwarfs and T Tauri stars in Cha I and that the differences found in the cumulative $R V$ distributions for both groups might be explained by systematic errors are both consistent with the finding of no mass dependence of the velocities in models by Bate et al. (2003), Bate \& Bonnell (2005) and with the finding of only small mass dependence in the model by Delgado-Donate et al. (2004). However, the observed $R V$ dispersions in Cha I for both the brown dwarfs and the stars are much smaller than predicted by these models. There is a difference of about a factor of ten to one hundred in stellar density between Cha I and the simulated star-forming regions. While an extrapolation of the predictions of Bate \& Bonnell (2005) to these smaller densities might be consistent with the empirical value measured by us for Cha I, we show that such an extrapolation is not advised, amongst others, because it does not, at the same time, yield consistent results for the less dense Taurus region.

Sterzik \& Durisen (2003) and Umbreit et al. (2005) provide cumulative distributions of their results, which we can compare directly to our observed cumulative $R V$ distributions. The high-velocity tail seen by Sterzik \& Durisen (2003) is even more pronounced in the models by Umbreit et al. (2005), who consider ongoing accretion during the dynamical encounters. However, it is not seen in the observed $R V$ distribution of brown dwarfs in Cha I. We suggest that the brown dwarfs in Cha I show no high-velocity tail, but the other possibility is that we have missed the 40-50\% fast-moving brown dwarfs in our relatively small sample comprised of nine objects.

We found that a subsample of known predominantly wide binaries among the $\mathrm{T}$ Tauri stars studied in Cha I has (i) a smaller $R V$ dispersion $\left(1.0 \mathrm{~km} \mathrm{~s}^{-1}\right)$ and (ii) no high-velocity tail compared to the remaining "single" $\mathrm{T}$ Tauri stars $(R V$ dispersion $1.4 \mathrm{~km} \mathrm{~s}^{-1}$ ). This observational hint of a kinematic difference between singles and binaries is in line with theoretical predictions by Sterzik \& Durisen (2003).

The comparison of observations in Cha I with theoretical calculations had to deal with the difficulty that the current models do not predict uniform quantities to describe the velocity distribution. Furthermore, at the moment, their predictive power is limited by simplifications adopted therein, e.g. the lack of gravitational potential in $\mathrm{N}$-body simulations and the lack of feedback processes in hydrodynamical calculations, as well as the fact that the latter are performed for much higher densities than found in intensively observed clouds like Cha I or Taurus.

The observational constraint for the velocity distribution of a homogeneous group of closely confined very young brown dwarfs provided by the high-resolution spectroscopic study here is a first empirical upper limit for ejection velocities. It would be valuable to extent these observations to those not yet observed and/or to newly detected young brown dwarfs in Cha I (e.g. López Martíet al. 2004) and in other star-forming regions, in order to put the results on an improved statistical basis. 
Acknowledgements. I am grateful to the referee, Fernando Comerón, for very helpful comments which significantly improved the paper. I would also like to thank Kengo Tachihara, Pavel Kroupa, Matthew Bate, Eduardo Delgado-Donate, and Stefan Umbreit for interesting discussions and Michael C. Liu for hinting at double entries in a table in a previous publication. It is a pleasure to acknowledge the excellent work of the ESO staff at Paranal, who took all the UVES observations the present work is based on in service mode. Furthermore, I acknowledge support by a Marie Curie Fellowship of the European Community program "Structuring the European Research Area" under contract number FP6-501875. This research made use of the SIMBAD database, operated at CDS, Strasbourg, France.

\section{Appendix A: Details on the T Tauri star sample}

The sample of T Tauri stars was revised in the presented work compared to Joergens \& Guenther (2001) by identifying five double entries under different names that correspond to the very same objects in their Table 2 ( $\mathrm{Sz} 9 \equiv \mathrm{CS}$ Cha; Sz 11 ECT Cha; Sz $36 \equiv$ WY Cha; Sz $41 \equiv$ RX J1112.7-7637; Sz 42 $\equiv$ CV Cha). Furthermore, two stars (Sz15/T19 and B33/CHXR25) of the previous $\mathrm{T}$ Tauri star sample were revealed as foreground stars by Luhman (2004) and were rejected. Moreover, $R V$ measurements by Walter (1992) of several T Tauri stars in Cha I were not considered for the previous kinematic study and were taken into account for the revised version presented here. For CHX18N, the $R V$ measured by Walter (1992) is significantly discrepant with the measurement of Covino et al. (1997), thus hinting at a long-period spectroscopic binary. For the earlier publication, only the measurement by Covino et al. (1997) of $19.0 \pm 2.0 \mathrm{~km} \mathrm{~s}^{-1}$ for this star was taken into account, which made it an outlier in the T Tauri star sample, while the paper on hand also takes the previously overlooked $R V$ determination of $13 \pm 2 \mathrm{~km} \mathrm{~s}^{-1}$ by Walter (1992) into account. Therefore, the new mean $R V$ for this object results in a narrower $R V$ dispersion of the whole sample.

The kinematic study of $\mathrm{T}$ Tauri stars was also revised by an improved analysis of the UVES spectra for B34, CHXR74, and $\mathrm{Sz} 23$ and, in addition to this, by taking into account new UVES-based $R V \mathrm{~s}$ for CHXR74 and Sz23 obtained by us in 2002 and 2004. For $\mathrm{Sz} 23$, the change in $R V$ from 2000 to 2004 is marginal, whereas for CHXR 74, the discrepancy between the mean $R V$ for 2000 and for 2004 is more than $2 \mathrm{~km} \mathrm{~s}^{-1}$, hinting at a spectroscopic companion (cf. Joergens 2003, 2006).

\section{References}

Armitage, P. J., \& Clarke, C. J. 1997, MNRAS, 285, 540

Bate, M. R., Bonnell, I. A., \& Bromm, V. 2002, MNRAS, 332, L65

Bate, M. R., Bonnell, I. A., \& Bromm, V. 2003, MNRAS, 339, 577
Bate, M. R., \& Bonnell, I. A. 2005, MNRA,S 356, 1201

Brandner, W., Alcalá, J. M., Kunkel, M., Moneti, A., \& Zinnecker, H. 1996, A\&A, 307, 121

Brandner, W., \& Zinnecker, H. 1997, A\&A, 321, 220

Comerón, F., Rieke, G. H., \& Neuhäuser, R. 1999, A\&A, 343, 477

Comerón, F., Neuhäuser, R., \& Kaas, A. A. 2000, A\&A, 359, 269

Covino, E., Alcalá, J. M, Allain, S., et al. 1997, A\&A, 328, 187

Dekker, H., D’Odorico, S., Kaufer, A., Delabre, B., \& Kotzlowski, H. 2000, in SPIE 4008, 534, ed. M. Iye, \& A. Moorwood

Delgado-Donate, E. J., Clarke, C. J., \& Bate, M. R. 2003, MNRAS, 342,926

Delgado-Donate, E. J., Clarke, C. J., \& Bate, M. R. 2004, MNRAS, 347, 759

Dubath, P., Reipurth, B., \& Mayor, M. 1996, A\&A, 308, 107

Durisen, R. H., Sterzik, M. F., \& Pickett, B. K. 2001, A\&A, 371, 952

Ghez, A. M., McCarthy, D. W., Patience, J. L., \& Beck, T. L. 1997, ApJ, 481, 378

Hartmann, L., Hewett, R., Stahler, S., \& Mathieu, R. D. 1986, ApJ, 309,275

Joergens, V., \& Guenther, E. 2001, A\&A, 379, L9

Joergens, V. 2003, Ph.D. Thesis, Ludwigs-Maximilians Universität München

Joergens, V., Neuhäuser, R., Guenther, E. W., Fernández, M., \& Comerón, F. 2003, in Brown Dwarfs, ed. E. L. Martín, Astronomical Society of the Pacific (San Francisco: ASP), IAU Symp., 211, 233

Joergens, V. 2005, in Rev. Mod. Astron., ed. S. Roeser (Weinheim: Wiley), 18, 216

Joergens, V. 2006, A\&A, 446, 1165

Kroupa, P., \& Bouvier, J. 2003a, MNRAS, 346, 343

Kroupa, P., \& Bouvier, J. 2003b, MNRAS, 346, 369

López Martí, B., Eislöffel, J., Scholz, A., \& Mundt, R. 2004, A\&A, 416,555

Luhman, K. 2004, ApJ, 602, 816

Marcy, G., Butler, P., Vogt, S., et al. 2005, ApJ, 619, 570

Moutou, C., Mayor, M., Bouchy, F., et al. 2005, A\&A, 439, 367

Mizuno, A., Hayakawa, T., Tachihara, K., et al. 1999, PASJ, 51, 859

Neuhäuser, R., \& Comerón, F. 1998, Science, 282, 83

Neuhäuser, R., \& Comerón, F. 1999, A\&A, 350, 612

Onishi, T., Mizuno, A., Kawamura, A., Ogawa, H., \& Fukui, Y. 1996, ApJ, 465, 815

Reipurth, B., \& Zinnecker, H. 1993, A\&A, 278, 81

Reipurth, B., \& Clarke, C. 2001, ApJ, 122, 432

Sterzik, M. F., \& Durisen, R. H. 1995, A\&A, 304, L9

Sterzik, M. F., \& Durisen, R. H. 1998, A\&A, 339, 95

Sterzik, M. F., \& Durisen, R. H. 2003, A\&A, 400, 1031

Tachihara, K., Onishi, T., Mizuno, A., \& Fukui, Y. 2002, A\&A, 385, 909

Umbreit, S., Burkert, A., Henning, T., Mikkola, S., \& Spurzem, R. 2005, ApJ, 623, 940

Valtonen, M., \& Mikkola, S. 1991, ARA\&A, 29, 9

Walter, F. M. 1992, AJ, 104, 758

White, R. J., \& Basri, G. 2003, ApJ, 582, 1109 